\title{
On the Apparent Relationship Between Total Solar Irradiance and the Atmospheric Temperature at 1 Bar on Three Terrestrial-type Bodies
}

\author{
Robert Ian Holmes \\ Science \& Engineering Faculty, Federation University, Ballarat, Australia
}

Email address:

r.holmes@federation.edu.au

To cite this article:

Robert Ian Holmes. On the Apparent Relationship Between Total Solar Irradiance and the Atmospheric Temperature at 1 Bar on Three Terrestrial-type Bodies. Earth Sciences. Vol. 8, No. 6, 2019, pp. 346-351. doi: 10.11648/j.earth.20190806.15

Received: October 23, 2019; Accepted: December 18, 2019; Published: December 26, 2019

\begin{abstract}
It has been discovered that there appears to exist a close relationship between relative differences in total solar irradiance and the atmospheric temperature, at a pressure of $1 \mathrm{bar}$, on all three terrestrial-type bodies which possess thick atmospheres. The apparent relationship is through the quaternary root of total solar irradiance at $1 \mathrm{bar}$, and applies to the planetary bodies Venus, Earth and Titan. The relationship is so close that the average surface atmospheric temperature of Earth can be easily calculated to within 1 Kelvin $(0.5 \%)$ of the correct figure by the knowledge of only two numbers, neither of which are related to the Earth's atmosphere. These are; the atmospheric temperature in the Venusian atmosphere at 1 bar, and the top-of-atmosphere solar insolation of the two planets. A similar relationship in atmospheric temperatures is found to exist, through insolation differences alone, between the atmospheric temperatures at 1 bar of the planetary bodies Titan and Earth, and Venus and Titan. This relationship exists despite the widely varying atmospheric greenhouse gas content, and the widely varying albedos of the three planetary bodies. This result is consistent with previous research with regards to atmospheric temperatures and their relationship to the molar mass version of the ideal gas law, in that this work also points to a climate sensitivity to $\mathrm{CO}_{2}$ - or to any other 'greenhouse' gas - which is close to or at zero. It is more confirmation that the main determinants of atmospheric temperatures in the regions of terrestrial planetary atmospheres which are $>0.1$ bar, is overwhelmingly the result of two factors; solar insolation and atmospheric pressure. There appears to be no measurable, or what may be better termed 'anomalous' warming input from a class of gases which have up until the present, been incorrectly labelled as 'greenhouse' gases.
\end{abstract}

Keywords: Climate Change, Global Climate Change, Global Warming, Greenhouse Gases, Greenhouse Effect, Venus Temperature, Earth Temperature, Titan Temperature, Atmospheric Thermal Gradient

\section{Introduction}

It is known that all planetary bodies with thicker atmospheres naturally set up a rising thermal gradient in that part of the atmosphere, which is higher than a pressure of 0.1 bar, until that bodies' surface is reached [1]. Previous works $[2-4,14]$ have indicated that there may be a relationship between total solar irradiance (TSI), atmospheric pressures and planetary atmospheric temperature on bodies which possess thick atmospheres. It is shown here that this relationship appears to exist across all three terrestrial-type bodies which possess thick atmospheres. If this relationship proves to be robust, then it will present difficulties for several current hypothesis with regards to what forms and causes planetary atmospheric temperature. In particular, the idea that greenhouse gases play a significant role in forming atmospheric temperature, (15) and the idea that albedo plays a significant role in forming atmospheric temperatures (22).

\subsection{A Physical Law Must Be Universal}

Whenever a hypothesis is used to explain the Earth's temperature, it must also take into account the universality of the physical laws of nature. For instance, it must explain how a similar gradient/enhancement appears in other planetary atmospheres with widely varying levels of greenhouse gases [1]. And must also explain, as shown here, how the 
atmospheric temperature at 1 bar, in all three terrestrial-type bodies which possess thick atmospheres seem to be related to the quaternary root of relative differences in total irradiation at equal pressure, regardless of the very different albedos and greenhouse gas percentages of these bodies.

\subsection{Applying the Stefan-Boltzmann Law of Isolated Bodies to Planets with Atmospheres}

It is known from the Stefan-Boltzmann law that the radiating temperature of an isolated planetary body in space, (one which possesses no atmosphere), varies with the fourthroot of the power incident upon it [30]. And given that previous works [1-4] have detailed that a principle factor in determining atmospheric temperatures on planetary bodies with thick atmospheres is atmospheric pressure, logic dictates that these may be combined - initially at a standard for pressure - for example, 1 bar.

\section{Calculated vs Measured Temperatures of the Three Planetary Bodies at 1 bar}

If this hypothesised relationship proves to be true, and the atmospheric temperature of a planetary body in space varies with the quaternary root of relative TSI difference, it will mean that the temperature of Venus at 1atm (Tv) should be found to be the quaternary root of 1.91 times the temperature on Earth at 1atm (Te). Note that Venus receives 1.91 times the solar insolation of Earth [5].

For Titan, the only other terrestrial-type body with a thick atmosphere, again; it will mean that the temperature of Titan at $1 \mathrm{~atm}(\mathrm{Tt})$ should be the quaternary root of 0.01089 times the temperature on Earth at 1atm (Te). Note that Titan receives 0.01089 times the solar insolation of Earth [5].
Therefore;

$$
\text { Tplanet } 1 \text { bar }=\sqrt[4]{\text { TSI relative }} \times \text { Te }
$$

Earth

$$
\begin{aligned}
& \mathrm{Tv}=\sqrt[4]{1.91} \times \mathrm{Te} \\
& \mathrm{Tv}=1.176 \times 288 \\
& \mathrm{Tv}=339 \text { Kelvin }
\end{aligned}
$$

Earth

$$
\begin{aligned}
\mathrm{Te} & =\sqrt[4]{0.523} \times \mathrm{Tv} \\
\mathrm{Te} & =0.850 \times 340 \\
\mathrm{Te} & =289.1 \text { Kelvin }
\end{aligned}
$$

Titan

$$
\begin{gathered}
\mathrm{Tt}=\sqrt[4]{0.01089} \times \mathrm{Te} \\
\mathrm{Tt}=0.323 \times 288 \\
\mathrm{Tt}=93 \text { Kelvin }
\end{gathered}
$$

\subsection{The Disappearance of 'Albedo' and the 'Greenhouse'

\begin{tabular}{|c|c|c|c|c|c|c|}
\hline Planet & Measured Temp & Relative TSI & Fourth Root TSI & Calculated Temp & Albedo & GHG \% \\
\hline Venus & 340 Kelvin & 1.91 & 1.176 & 339 Kelvin & $77 \%$ & $96.5 \%$ \\
\hline Earth & 288 Kelvin & 0.523 & 0.850 & 289.1 Kelvin & $30 \%$ & $2.5 \%$ \\
\hline Titan & 85-90 Kelvin & 0.01089 & 0.323 & 93 Kelvin & $22 \%$ & $2.7 \%$ \\
\hline
\end{tabular} Gas Effect}

Logic perhaps dictates that the widely differing albedos and 'greenhouse' gas content must mean something for planetary temperatures. Yet the temperatures at 1 bar, calculated from other planets, using relative TSI alone, are surprisingly close to the measured temperatures; it is insufficient to simply say they are wrong. Why and how they are wrong will need to be comprehensively explained.

Table 1. The calculated vs measured temperature of three terrestrial-type bodies at 1 atm.

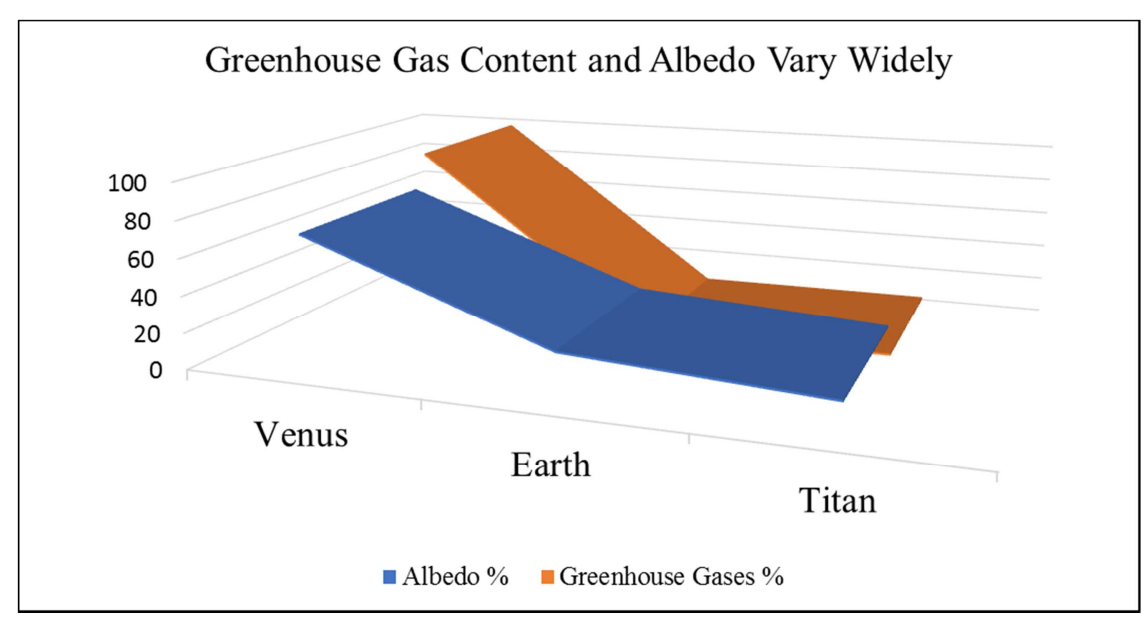

Figure 1. Greenhouse gas content and albedo vary widely across Venus, Earth and Titan. 


\subsection{Venusian \& Titan Temperature at 1atm Is Accurately Predicted by Relative TSI Alone}

The Venusian lapse rate, perhaps surprisingly considering the very different atmospheric conditions, is very similar to Earth's at $7.7 \mathrm{~K} / \mathrm{km}$ but extends much higher, up to at least $50 \mathrm{~km}$ [6]. A little below that height at $49 \mathrm{~km}$ is where a pressure of $1 \mathrm{~atm}$ is to be found and is where a temperature of $\sim 340 \mathrm{~K}$ has been measured [6-8] to prevail. The measured temperature in the Venusian atmosphere cited here comes from Venera's 8, 9, 10, 11 and 12 and from the Pioneer Sounder at $1 \mathrm{~atm}$, averages $340 \mathrm{~K}[12,13,20]$. Titan data comes from NASA, occultations and the Huygens lander [5, $10,11,23,31,32]$. Earth data $[5,9,16]$. The temperature at $1 \mathrm{~atm}$ on Venus, divided by the fourth-root of the insolation difference, results in $289 \mathrm{~K}$ - a value very close to Earth's average surface temperature of $288 \mathrm{~K}$ at $1 \mathrm{~atm}$. Yet Venus has a $96.5 \%$ greenhouse gas atmosphere, compared to Earth's at just $2.5 \%$. It's hard to imagine atmospheres with such a differing greenhouse gas content, yet there still remain very strong similarities in the rate of the tropospheric thermal gradient and as seen here, in the relative insolation-adjusted temperatures at $1 \mathrm{~atm}$. These measurements, relationships and the similarity of the thermal gradients point strongly towards the existence of a universal physical law which governs planetary atmospheric temperatures - and one which does not take into account the relative greenhouse gas contents; instead, this law clearly operates as if greenhouse gases are not special.

This result is consistent with previous research [3] with regards to atmospheric temperatures and their relationship to the molar mass version of the ideal gas law, in that this work also points to a climate sensitivity to $\mathrm{CO}_{2}$ - or to any other so-called 'greenhouse' gas - which is close to or at zero. It is more confirmation that the main determinants of atmospheric temperatures in the regions of terrestrial planetary atmospheres which are $>0.1$ bar, is overwhelmingly the result of two factors; solar insolation and atmospheric pressure. There appears to be no measurable, or what may be better termed 'anomalous' warming or cooling input in these regions from a class of gases which have up until the present, been apparently incorrectly labelled as 'greenhouse' gases. 'Anomalous' meaning an effect outside of the contributions from their three basic properties of density, pressure and molar mass; in short, as far as it is possible to measure, there is such thing as a special class of 'greenhouse' gases.

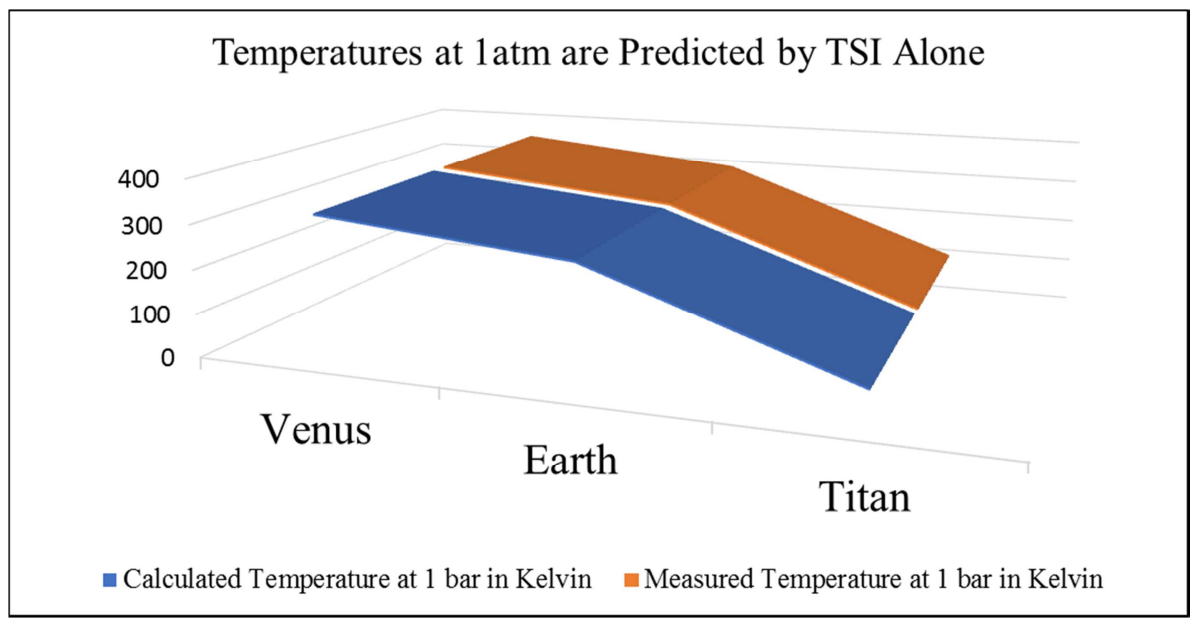

Figure 2. Temperatures at 1 atm are accurately predicted by TSI alone across these three bodies.

\section{Implications for the 'Greenhouse Effect' of 'Greenhouse' Gases}

If this relationship between TSI alone and planetary temperatures at 1 bar proves to be real, it will have important implications for the very existence of the socalled 'greenhouse effect' as it has been proposed by the intergovernmental panel on climate change and others [15, $19,21,22]$. The data shows that the 'greenhouse gas' concentration varies widely from the low $2.7 \%$ and $2.5 \%$ [5, 9-11] for Titan and Earth respectively, to the very high $96.5 \%$ for Venus; the implication must be that there cannot be any special warming effect from the so-called 'greenhouse' gases. This result adds and contributes to considerable other evidence $[2-4,14,17,18,24-29]$, that there is no sign of any 'greenhouse effect' from 'greenhouse' gases on any of these three bodies. TSI and a thermal gradient / thermal enhancement set up by auto-compression [2, 3] and convection alone appear to be the main drivers which establish planetary atmospheric temperatures on these bodies.

Additionally, if the quaternary root of relative differences in TSI at the equal atmospheric pressure of $1 \mathrm{~atm}$ does indeed predict temperature irrespective of albedo, then the means by which planetary atmospheric temperatures are presently calculated, which include albedo, will have to be revised.

\subsection{Average Temperature of Earth's Surface Can Be Accurately Calculated-from Venus}

The relationship between a resultant atmospheric temperature at $1 \mathrm{bar}$ and the atmospheric pressure / relative 
TSI combination means that Earth's average surface temperature can be easily and accurately calculated by measuring just two input factors; the temperature of the Venus atmosphere at $1 \mathrm{bar}$, and the relative distances of these planets from the Sun (i.e. the relative TSI of Earth and Venus).

Thus;

$$
\begin{gathered}
\text { Tplanet } 1 \text { bar }=\sqrt[4]{\text { TSI relative }} \times \text { Te } \\
\text { Te }=\frac{\text { Tvenus at } 1 \text { bar }}{\sqrt[4]{\text { TSI relative }}} \\
\text { Te }=\frac{340}{\sqrt[4]{1.91}}
\end{gathered}
$$

$\mathrm{Te}=289.2$ Kelvin

This temperature of the Earth's surface, derived from Venus, is within $0.5 \%$ of the correct figure. The same calculation - using Titan's atmospheric temperature at 1 bar and its relative TSI, to arrive at an estimate of the Earth's surface temperature, is not quite as accurate as this but is still within a few percent of the correct temperature. The reason for the discrepancy could be due to Titan's atmospheric temperature at 1 bar being measured less often and being less well known than the Venusian atmospheric temperatures. However, it could not be due to an anomalous effect from Titan's 'greenhouse' gas content, unless those 'greenhouse' gases somehow cause a slight cooling. Note that Titan has a far greater atmospheric greenhouse gas content (in global warming potential terms) $[10,31,32]$ than Earth has.

A calculation from formula 1, using Earth or Venus as inputs, results in a predicted Titan 1 bar temperature of 93 Kelvin. The surface temperature on Titan, measured by the Cassini probe has been shown to vary between 90 Kelvin at the poles and 94 Kelvin at the equator $(31,32)$. However, the surface pressure on Titan is 1.45 bar; and given the thermal gradient known to exist there, [1] the temperature at 1 bar is expected to be somewhat lower, in the range 85 - 90 Kelvin. An investigation into the reasons for this slight discrepancy between predicted and measured temperatures at 1 bar is outside the scope of this work.

\subsection{Predictions of Atmospheric Temperatures Become Possible}

A further consequence - again if this simple relationship holds between relative TSI and atmospheric temperatures at 1 atm - is that atmospheric temperature predictions become possible. For example, a prediction of the 1 atm temperature of Earth at double (Td) its current distance from the Sun can easily be made;

$$
\begin{gathered}
\text { Earth } \times 2 \mathrm{Td}=\sqrt[4]{0.250} \times \mathrm{Te} \\
\mathrm{Td}=0.707 \times 288 \\
\mathrm{Td}=204 \text { Kelvin }
\end{gathered}
$$

\section{Consistent with Previous Research}

The insolation-related correlation in temperature data from $1 \mathrm{~atm}$ points towards there being no significant (or anomalous) warming from the 'greenhouse' gases present and is consistent with previous [3, 4, 14, 29] research. The previous work by Holmes [3] reveals that a 'greenhouse effect' caused by 'greenhouse gases' effectively does not exist in planetary troposphere's (the regions of terrestrial atmospheres which are $>10 \mathrm{kPa}$ ). A thermal gradient and a surface thermal enhancement do exist - but these are attributed to autocompression / convection and not specifically to any anomalous input from 'greenhouse' gases. The final conclusions of that research can be summarised thus;

Postulates for this exercise;

a) The Ideal Gas Law is correct.

b) The same external conditions such as insolation and auto-compression prevail.

\subsection{Why the Ideal Gas Law Is Inconsistent with Anomalous Warming from a Greenhouse Gas Effect}

For a 'greenhouse effect' caused by 'greenhouse gases' to occur in a convecting atmosphere (one of $>10 \mathrm{kPa}$ ), a large anomalous change must happen in the density, the pressure or both. No anomalous changes of this magnitude have been detected in any planetary atmospheres. In fact, anomalous changes of this nature are forbidden by the ideal gas law and its derivatives like the molar mass version, because they treat all gases equally. The molar mass version of the ideal gas law accurately determines - and allows - an atmospheric temperature to be determined based only on a gas constant and three gas properties; namely pressure, density and molar mass. No reference to the radiative properties of a gas are needed or included. Therefore, it can be said that different concentrations of gases at the same or at different times can provide the same temperature or different temperatures.

However - the same concentrations of gases cannot provide different temperatures at different times.

The formula $\mathrm{T}=\mathrm{P} \mathrm{M} / \mathrm{R} \rho$ which is derived from the ideal gas law, forbids it. This fact presents a terminal conflict with the greenhouse gas hypothesis, as it is presented by the IPCC* [15].

\subsection{Why There Is a Terminal Conflict Between the Ideal Gas Law and the IPCC's Reports}

*The reason for the terminal conflict is because it is stated in all IPCC reports that there exists a time delay to reach 'equilibration', due to the nature of the greenhouse gas effect resulting in what the IPCC calls the ECS (Equilibrium Climate Sensitivity) climate sensitivity to $\mathrm{CO}_{2}$ being in the range of $1.5 \mathrm{C}-4.5 \mathrm{C}$. The IPCC reports state that if there was a sudden doubling in the atmospheric greenhouse gas $\mathrm{CO}_{2}$, the greenhouse gas effect from this would operate slowly, causing an eventual $\sim 3 \mathrm{c}$ of warming over centuries to millennia.

Therefore, the IPCC's claim is that since the IPCC's climate sensitivity range is $1.5 \mathrm{C}-4.5 \mathrm{C}$, the temperature must 
rise significantly over time, with the same prevailing atmospheric gas concentrations, and there would be no rapid equilibration, as the ideal gas law and it's derivative, the molar mass version, demand. This represents the terminal conflict between the IPCC's description of the 'greenhouse gas effect' and way the molar mass version of the ideal gas law operates. The ideal gas law is a pillar of gas thermodynamics and physics and cannot be lightly discounted in favor of a wholly hypothetical warming from 'greenhouse gases' such as $\mathrm{CO}_{2}$, which has never actually been empirically detected or quantified in the real atmosphere.

\section{Conclusion}

The temperature of the atmospheres at 1 bar $(101.3 \mathrm{kPa})$ of all three of the terrestrial-type planetary bodies with thick atmospheres, despite the large differences between them both in atmospheric greenhouse gas content and albedo (Figure 1), appears to relate almost exclusively to the quaternary root of relative differences in TSI (Figure 2). This seems to point to the main determinants of planetary atmospheric temperatures of terrestrial-type bodies which possess thick atmospheres, being atmospheric pressure and TSI, not albedo and greenhouse gas content. If this relationship proves to be a real feature of planetary atmospheric physics, it will have farreaching effects for how albedo and 'greenhouse' gas content are treated when calculating atmospheric temperatures in the future. The relationship tends to add to previous work that indicates the likelihood of a very low or a zero, climate sensitivity for $\mathrm{CO}_{2}$ [2-4,14,17,18,24-29].

\section{References}

[1] Robinson, T. D., \& Catling, D. C. (2014). Common 0.1 [thinsp] bar tropopause in thick atmospheres set by pressure-dependent infrared transparency. Nature Geoscience, 7 (1), 12-15.

[2] Holmes, R. I. (2017c). Molar Mass Version of the Ideal Gas Law Points to a Very Low Climate Sensitivity. Earth Sciences $6(6), 157$.

[3] Holmes, R. I. (2018). Thermal Enhancement on Planetary Bodies and the Relevance of the Molar Mass Version of the IGL to the Null Hypothesis of Climate Change. Earth, 7 (3), 107-123.

[4] Nikolov, N., \& Zeller, K. (2017). New insights on the physical nature of the atmospheric greenhouse effect deduced from an empirical planetary temperature model. Environment Pollution and Climate Change, 1 (2), 112.

[5] NASA fact sheet data on the planets, (2017). Accessed 19/9/19. https://nssdc.gsfc.nasa. gov/planetary/planetfact.html

[6] Seiff, A. (1983). 11. Thermal Structure of the Atmosphere of Venus. Venus, 215.

[7] Pätzold, M., Häusler, B., Bird, M. K., Tellmann, S., Mattei, R., Asmar, S. W.,... \& Tyler, G. L. (2007). The structure of Venus' middle atmosphere and ionosphere. Nature, 450 (7170), 657.
[8] Zasova, L. V., Ignatiev, N., Khatuntsev, I., \& Linkin, V. (2007) Structure of the Venus atmosphere. Planetary and Space Science, 55 (12), 1712-1728.

[9] Wikipedia, Properties of Earth's atmosphere, (2017). Accessed 19/9/19. https://en.wiki pedia.org/wiki/Density_of_air

[10] Fulchignoni, M., Ferri, F., Angrilli, F., Ball, A. J., Bar-Nun, A., Barucci, M. A.,... \& Coradini,, M. (2005). In situ measurements of the physical characteristics of Titan's environment. Nature, 438 (7069), 785-791.

[11] Lindal, G. F., Wood, G., Hotz, H., Sweetnam, D., Eshleman, V., \& Tyler, G. (1983). The atmosphere of Titan: An analysis of the Voyager 1 radio occultation measurements. Icarus, 53 (2), 348-363.

[12] Moroz, V., Ekonomov, A., Moshkin, B., Revercomb, H., Sromovsky, L., Schofield, J. Tomasko, M. G. (1985). Solar and thermal radiation in the Venus atmosphere. Advances in Space Research, 5 (11), 197-232.

[13] Zasova, L., Ignatiev, N., Khatuntsev, I., \& Linkin, V. (2007). Structure of the Venus atmosphere. Planetary and Space Science, 55 (12), 1712-1728.

[14] Jelbring, H. (2003). The "Greenhouse Effect" as a Function of Atmospheric Mass. Energy \& Environment, 14 (2), 351-356

[15] Team, C. W., Pachauri, R., \& Meyer, L. (2014). IPCC, 2014: Climate Change 2014: Synthesis Report. Contribution of Working Groups I. II and III to the Fifth Assessment Report of the Intergovernmental Panel on Climate Change. IPCC, Geneva, Switzerland, 151.

[16] Stephens, G. L., O'Brien, D., Webster, P. J., Pilewski, P., Kato, S., \& Li, J.-1. (2015). The albedo of Earth. Reviews of Geophysics, 53 (1), 141-163.

[17] Lüdecke, H.-J., Hempelmann, A., \& Weiss, C. (2013). Multiperiodic climate dynamics: spectral analysis of long-term instrumental and proxy temperature records. Climate of the Past, 9 (1), 447.

[18] Cederlöf, M. (2014). Using seasonal variations to estimate Earth's response to radiative forcing. http://www.klimatupplysningen.se/wpcontent/uploads/2014/11/seasonal_variations_0_5.pdf

[19] Lacis, A. A., Schmidt, G. A., Rind, D., \& Ruedy, R. A. (2010). Atmospheric CO: Principal control knob governing Earth's temperature. Science, 330 (6002), 356-359.

[20] Jennings, D. E., Cottini, V., Nixon, C. A., Flasar, F. M., Kunde, V. G., Samuelson, R. E.,... \& Coustenis, A. (2011). Seasonal changes in Titan's surface temperatures. The Astrophysical Journal Letters, 737 (1), L15.

[21] Crisp, D. (2007). Greenhouse Effect and Radiative Balance on Earth and Venus. Presentation to the Venus Exploration Assessment Group (VEXAG).

[22] Pierrehumbert, R. T. (2011, November). Infrared radiation and planetary temperature. In AIP Conference Proceedings (Vol. 1401, No. 1, pp. 232-244). AIP.

[23] Porco, C. C., Baker, E., Barbara, J., Beurle, K., Brahic, A., Burns, J. A.,... \& Denk, T. (2005). Imaging of Titan from the Cassini spacecraft. Nature, 434 (7030), 159.

[24] Fenton, L., Geissler, P., \& Haberle, R. (2006). Global warming on Mars. Paper presented at the AGU Fall Meeting Abstracts. 
[25] Sromovsky, L., Fry, P., Limaye, S., \& Baines, K. (2003). The nature of Neptune's increasing brightness: Evidence for a seasonal response. Icarus, 163 (1), 256-261

[26] Ravilious, K. (2007). Mars melt hints at solar, not human, cause for warming, scientist says. National Geographic News. http://news.nationalgeogr.../55741367.html.

[27] Pasachoff, J. M., Souza, S. P., Babcock, B. A., Ticehurst, D. R. Elliot, J., Person, M. Tholen, D. J. (2005). The structure of Pluto's atmosphere from the 2002 August 21 stellar occultation. The Astronomical Journal, 129 (3), 1718.

[28] Elliot, J. L., Person, M., Gulbis, A., Souza, S., Adams, E., Babcock, B.,... Pasachoff, J. (2007). Changes in Pluto's atmosphere: 1988-2006. The Astronomical Journal, 134 (1), 1.
[29] Lansner, F., \& Pedersen, J. O. P. (2018). Temperature trends with reduced impact of ocean air temperature, Energy \& Environment http://journals.sagepub.com/doi/10.1177/0958305X18756670

[30] Boltzmann, L. (1874). Sitzungsberichte der Kaiserlichen Akademie der Wissenschaften: Mathematischnaturwissenschaftlichen Klasse. Wien, 70, 275-300.

[31] Jennings, D. E., F. M. Flasar, V. G. Kunde, R. E. Samuelson, J. C. Pearl, C. A. Nixon, R. C. Carlson et al. "Titan's surface brightness temperatures." The Astrophysical Journal Letters 691, no. 2 (2009): L103. 